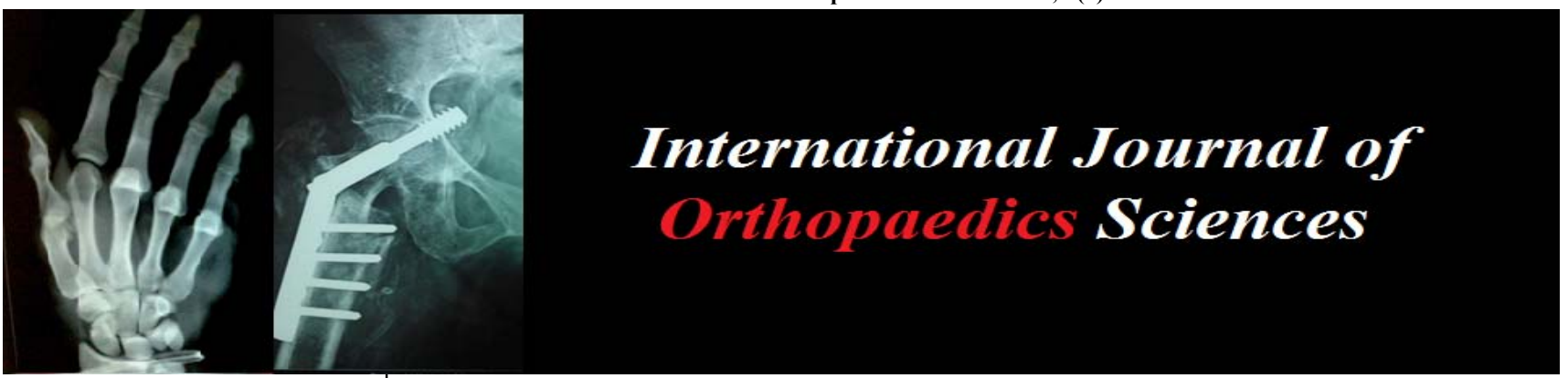

E-ISSN: 2395-1958

P-ISSN: 2706-6630

IJOS 2021; 7(2): 684-688

(C) 2021 IJOS

www.orthopaper.com

Received: 15-02-2021

Accepted: 21-03-2021

Dr. TH Prakashappa

D. Ortho, MS ortho, Professor of Orthopaedics, Sanjay Gandhi

Institute of Trauma and

Orthopaedics Bangalore,

Karnataka, India

Dr. Shyamsundar Y

Junior Resident, Sanjay Gandhi

Institute of Trauma and

Orthopaedics Bangalore,

Karnataka, India

Dr. Yogananda Gali Hanumaih Junior Resident, Sanjay Gandhi Institute of Trauma and Orthopaedics Bangalore, Karnataka, India

Corresponding Author: Dr. Shyamsundar $\mathbf{Y}$ Junior Resident, Sanjay Gandhi Institute of Trauma and Orthopaedics Bangalore, Karnataka, India

\section{Prospective study of functional outcome in surgical management of intracapsular femur neck fracture treated using modular bipolar prosthesis in elderly}

\author{
Dr. TH Prakashappa, Dr. Shyamsundar Y and Dr. Yogananda Gali \\ Hanumaih
}

DOI: https://doi.org/10.22271/ortho.2021.v7.i2i.2692

Abstract

Background: Displaced femoral neck fractures are common in the elderly patient, it is predicted that, the number of hip fractures would triple by 2050, and these patients are subjected to an increased disability, morbidity, and mortality. The optimal treatment for displaced fracture neck of femur with best clinical outcome is still inconclusive and determined by the mobility and functional demands of the patient.

Materials and Methods: Thirty cases of fracture neck of femur in elderly patients above the age of 60 years treated by hemiarthroplasty using bipolar prosthesis between November 2018 to May 2020 were selected according to inclusion and exclusion criteria. Cases were followed up for 6 months and the short term functional results were analysed by using modified Harris hip score.

Results: out of 30 patients, most of the patients were in the age group of 60 to 70 years with mean average age of 68.9 years. Females were predominant, there were $36 \%$ excellent results and $43 \%$ good results.

Conclusion: Modular Bipolar hemiarthroplasty for fractures neck femur provides better range of movement, freedom from pain and more rapid return to unassisted activity with an acceptable complication rate. The end functional results depend on the age of the patient, associated co-morbidity and optimum post-operative rehabilitation. Early functional results are satisfactory.

Keywords: femur neck fracture, hemiarthroplasty, modular bipolar prosthesis, harris hip score

\section{Introduction}

Hip fractures in the elderly represent a major public health concern, account for a quarter of all fractures in patients aged 75 years and over ${ }^{[1,2]}$. It is predicted that, the number of hip fractures would triple by $2050^{[3]}$, and these patients are subjected to an increased disability, morbidity, and mortality ${ }^{[4]}$.

The optimal treatment for displaced fracture neck of femur with best clinical outcome is still inconclusive ${ }^{[5]}$. And determined by the mobility and functional demands of the patient, two widely accepted options, i.e. monopolar or bipolar hemiarthroplasty (HA) and total hip arthroplasty (THA) both with optional use of bone cement in elderly are superior to internal fixation in terms of mobility, reoperation and functional outcome ${ }^{[6]}$

Bipolar prosthesis over monopolar prosthesis have higher percentage of satisfactory results, less post-operative pain, greater range of movements, more rapid return to normal routine and reduced frequency of acetabular erosion ${ }^{[7,8]}$, on the other hand THR is associated with increased operative time, increased blood loss and increased risk of dislocation ${ }^{[9]}$

Hemiarthroplasty done with a modular bipolar prosthesis, has the advantage of converting to total hip arthroplasty in future by adding the acetabular cup and allowing changes in length and offset following cementation during operation ${ }^{[10]}$.

Conceptually, the modular hemiarthroplasty design provides the surgeon with multiple options to restore leg length and femoral offset with a range of stem sizes, with different offset options, and head sizes that allow for minor adjustments during surgery.

This study aims to assess the functional outcome of modular bipolar hemiarthroplasty in NOF fractures in elderly. 


\section{Methods and methodology}

This is a prospective study of 30 cases of fracture neck of femur in elderly patients above the age of 60 years treated by hemiarthroplasty using modular bipolar prosthesis which were admitted to SGITO, Bangalore, Karnataka between November 2018 to May 2020 were included after obtaining the permission from the Institutional Ethical Committee.

Patients with age group $>60 \mathrm{yrs}$ of either sex and ambulatory, patient with intracapsular femur neck fracture, neglected femur neck fracture more than 3-4 weeks old in elderly patients, ability to understand the content of the subject information / informed consent form and to be willing to participate in the clinical investigation were included patients $<60$ yrs. of age, compound femur neck fractures, pathological fractures, polytrauma patients, Not willing to give informed written consent.

Cases were followed up for 6 months and the short term functional results were analysed by using Harris hip score.

Surgical approach-southern moore's posterior approach to the hip After induction of either spinal or epidural anaesthesia the patient was placed on the lateral position on the operative table with the affected side facing up. A curved incision is taken from $8 \mathrm{~cm}$ distal to the posterior superior iliac spine, extended distally and laterally, parallel with fibers of gluteus maximus muscle to the posterior margin of the greater trochanter. The incision is then directed distally $5-8 \mathrm{cms}$ along the femoral shaft. The deep fascia is exposed and divided in line with the skin incision. By blunt dissection the fibers of the gluteus maximus are separated taking care not to disturb the superior gluteal vessels in the proximal part of the exposure. The gluteus maximum muscle is split and short external rotators are exposed. Stay sutures are applied to the short external rotators, and a tenotomy of the short external rotators is done close to their insertion on the inner surface of the Greater trochanter. The short external rotators are retracted to protect the sciatic nerve and expose the posterior hip capsule. The capsule is incised by a T-shaped incision, and the hip flexed, adducted and internally rotated to dislocate the hip joint. Using a head extractor and bone levers, head is delivered out of the acetabulum and the acetabulum is cleared of debris. The size of the extracted head is measured by measuring gauze, and the size of prosthesis is selected. Preparation of proximal femur, The neck is trimmed leaving $1 \mathrm{~cm}$ medial calcar, on which the shoulder of the prosthesis would eventually sit. The proximal femur was reamed with rasp, the length of the rasp corresponding to the stem of the prosthesis. The direction of the insertion of the rasp for was ascertained by using the lesser trochanter as a guide to achieve correct seating of the prosthesis in $10-15^{0}$ anteversion. Insertion of the modular bipolar prosthesis, the appropriate sized femoral head component and neck component selected intraoperatively. The final seating of the prosthesis is by gentle blows with the help of a mallet and the inserter. Adequate seating of the prosthesis on the calcar is visualized directly. The hip joint is reduced by gentle traction with external rotation of the hip and simultaneous manipulation of the head of the prosthesis into the acetabulum. The range of movement in all directions is checked by taking the joint through the whole range of movements. The stability of the prosthesis and its tendency to dislocate is checked by flexing and adducting the hip. The limb is kept in slight abduction and external rotation for suturing the wound. Great care is taken to achieve adequate closure of the posterior capsule and anatomical reattachment of the short external rotators. The rest of the wound is closed in layers over a suction drain placed beneath the gluteus maximus. Hemostasis is maintained throughout the procedure. In 10 patients $43 \mathrm{~mm}$ prostheses were used. Cemented stems were used in 19 cases and Uncemented in 11 cases. The advantage was that the rasp corresponded to the exact length and width of the prosthesis which prevented any additional broaching of the canal and subsequent loose seating of the prosthesis or fractures of the femur. The medullary canal was washed with normal saline. Cement was prepared and finger packing into the canal was done. The prosthesis was then inserted maintaining correct anteversion.

\section{Postoperative protocol}

All the patients who were operated were kept in supine position with the involved lower limb in $20-30^{\circ}$ abduction. The drain was removed between 24-48 hours depending on the amount of collection. Perioperative prophylaxis of Ceftriaxone-S given $1.5 \mathrm{~g}$ 8th hourly intravenous was continued for the first 5-7 days. The wound was inspected at the time of drain removal and at the time of suture removal. If however, there was soakage of the dressing or if patient had high fever the wound was inspected accordingly. Most of our study patients were mobilized in bed on day one of surgery and with weight bearing as tolerated using a walker, within the 72 hours postoperative period. Patients were initially advised to touch weight bearing and later advised progress to full weight bearing as tolerated. The sutures were removed between 10-14 days. The patients were examined before discharge for the evidence of any infection at the operated site.

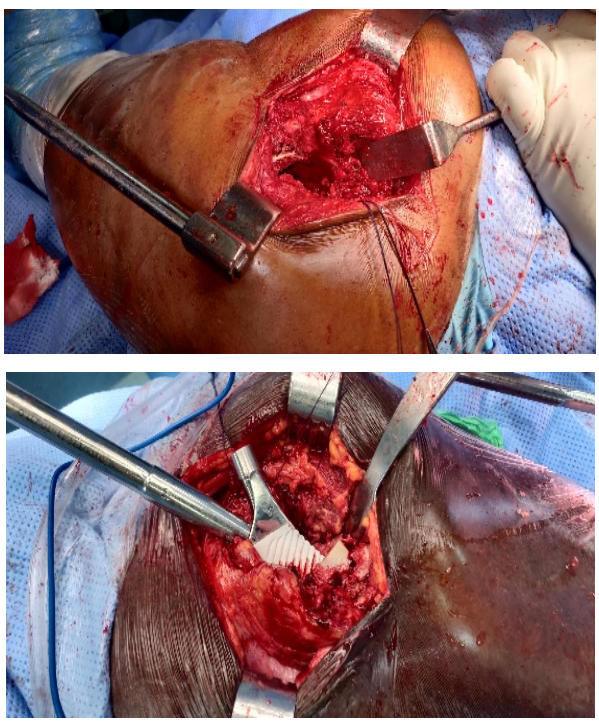

Fig 1: femoral canal preparation and insertion of modular femoral prosthesis

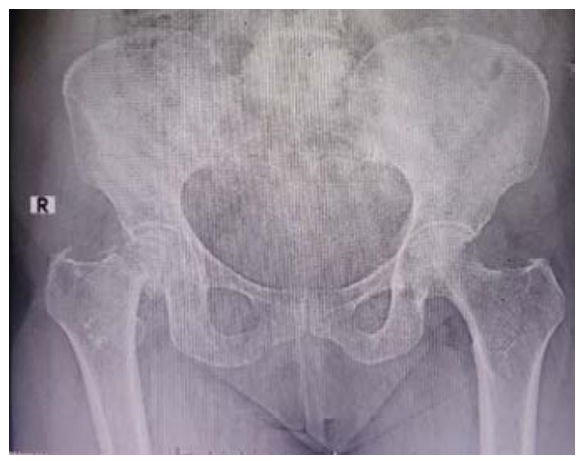

Fig 2: preop- radiograph 


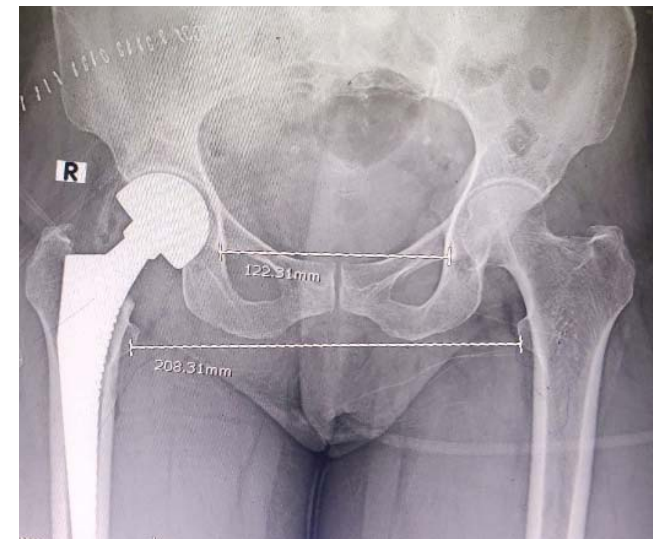

Fig 3: post -op radiograph

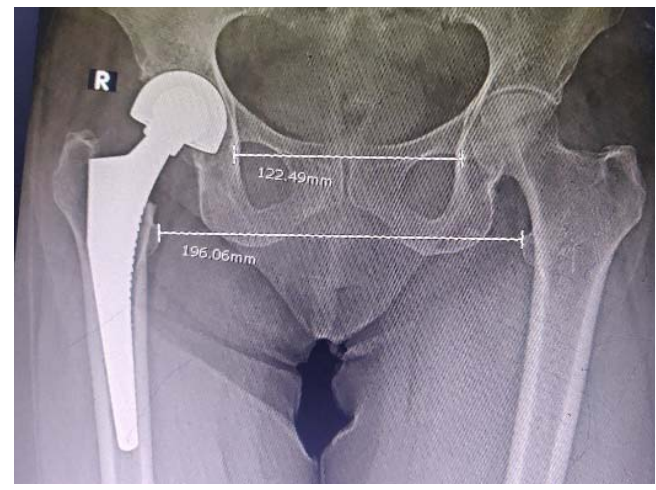

Fig 4: final followup

\section{Observations and Results}

The average age was noted to be 68.7 years.

Table 1: Age distribution

\begin{tabular}{|c|c|c|}
\hline Age & No.of patients & percentage \\
\hline $60-70$ & 21 & $70 \%$ \\
\hline $70-80$ & 7 & $23 \%$ \\
\hline$>80$ & 2 & $7 \%$ \\
\hline
\end{tabular}

Table 2: sex distribution

\begin{tabular}{|c|c|c|}
\hline Sex & No.of patients & Percentage \\
\hline Males & 9 & $30 \%$ \\
\hline Females & 21 & $70 \%$ \\
\hline
\end{tabular}

In 18 patients left side was involved and in 12 patients right side involved, Majority of the patients $(83.5 \%)$ had a transcervical fracture while four patients had sub-capital and one patient with basicervical fracture.

Table 3: Mode of Injury

\begin{tabular}{|c|c|c|}
\hline Mode of Injury & No. of patients & Percentage \\
\hline Slipping/trivial fall & 19 & $63.3 \%$ \\
\hline RTA & 11 & $36.6 \%$ \\
\hline
\end{tabular}

19 patients were operated with cemented hemiarthoplasty as these pateints had osteoporotic bone and to make the prosthesis snug fit to prevent iatrogenic fracture during serial rasping.

Table 5: Type of Hemiarthoplasty

\begin{tabular}{|c|c|c|}
\hline Type & No. of patients & Percentage \\
\hline Cemented & 19 & $63.3 \%$ \\
\hline uncemented & 11 & $36.6 \%$ \\
\hline
\end{tabular}

The size of the prostheses used, in $35 \%$ of the cases $45 \mathrm{~mm}$ prostheses were used. This was followed in frequency by 43 $\mathrm{mm}(27.5 \%), 47 \mathrm{~mm}(20 \%), 41 \mathrm{~mm}(10 \%)$ and $39 \mathrm{~mm}(2.5 \%)$ prostheses in the order of frequency. All the prostheses were inserted by press fit technique and $19(63.3 \%)$ required an additional augmentation with cement.

The pain status of the patient was assessed by Harris Hip score and showed that it decreases significantly following the operation. Only 2 patients had significant pain following the operation.

According to the clinical outcome of our study were graded excellent, good and fair. All the 30 patients before operation was categorized as poor according to Harris Hip score

Table 6: Final Harris hip score and clinical result

\begin{tabular}{|c|c|c|}
\hline HHS & No.of patients & percentage \\
\hline Excellent & 11 & $36.6 \%$ \\
\hline Good & 13 & $43.3 \%$ \\
\hline Fair & 4 & $13.3 \%$ \\
\hline Poor & 2 & $6.6 \%$ \\
\hline & 30 & 100 \\
\hline
\end{tabular}

Overall, 11 patients (36.6\%) achieved Excellent result, 13 patients $(43.3 \%)$ achieved Good result, 4 patients (13.3\%) achieved fair result and 2 patients $(6.6 \%)$ achieved poor result. $80 \%$ of the patients achieved an excellent or good result.

The progression of Harris hip score The average Harris Hip Score at 6 weeks after surgery was 60.04 with the highest score being 70 and the lowest being 43. The average Harris Hip Score at the second follow-up of 3 months was 73.46 with the maximum score being 86.8 and the minimum 59. At the final follow-up at 6 months the average Harris Hip Score was 82.7 with the highest being 94 and the lowest being 62 .

Comparing this score with preoperative score using paired' $t$ ' test was found to be statistically significant $(<0.05 \%)$.

In our study valgus malpositioning of the stem was found in 2 patients $(6.66 \%)$. There were no major postoperative complications like wound infection and other.

Limb lengthening $(<2 \mathrm{~cm})$ was observed in 5 patients $(16.6 \%)$ post- operatively due to technical errors in the form of the prosthesis sitting proud of the calcar.

There were no late postoperative complications like loosening, dislocation, erosion, secondary osteoarthritis, protrusion acetabuli, periprosthetic fracture till the final followup.

\section{Discussion}

In the present study of 30 cases of intracapsular fracture neck of femur treated with hemiarthroplasty with modular bipolar prosthesis of both the sexes. In all cases uncemented /cemented modular femoral prosthesis were used. All the study patients were taken up for the surgical procedure between the 4th and 12th day after the trauma, the average delay to surgery being 7 days. Delay in taking up for surgery was usually for optimizing the medical condition of the patient and some patients presented late. All cases were performed on an elective basis.

The mean age of the patients in the present study was 68.9 years. The aim of assessing age is to estimate the patient's mean survival time and their ability to comply with rehabilitation protocol. Patients with hip fractures have an increased mortality rate during the first year after fracture but after one year the mortality rate is comparable to that of the general population. The results of our study showed that age 
of the patient had minimal influence on the final clinical result. As in most standard studies, the present study also had a higher number of females with the left side more commonly affected than the right.

Majority of the patients $(83.5 \%)$ had a trans-cervical fracture while four patients had sub-capital and one patient with basicervical fracture. The anatomical type of fracture and the displacement did not have any effect on the final function.

The main difficulty faced was calculating the angle of the neck osteotomy which resulted in poor seating of the prosthesis collar on the neck and calcar. The second difficulty encountered was miscalculation of the amount of neck to be resected. Inadequate resection of the neck resulted in the prosthesis sitting proud of the calcar and subsequent limb lengthening in some cases.

Pain following hemiarthroplasty is a major concern. Bhandari et al. ${ }^{[13]}$ showed arthroplasty was a good option with regard to provision of pain relief with a relative risk of no or little pain of 1.12 (95\%CI,0.88 to 1.35$)$. Keating et al. ${ }^{[14]}$ showed significant decrease of pain out of 102 patients at 12-month follow-up.

In this study pain was reduced in 28 (93.33\%) patients which was statistically significant and comparable to other studies.

Ozer et al. ${ }^{[14]}$ showed $47 \%$ excellent result, $23 \%$ good result, $18 \%$ fair and $12 \%$ poor result treated with modular prosthesis in fracture neck of femur. Ali ${ }^{[11]}$ showed $75 \%$ satisfactory results out of 13 patients of fracture neck of femur treated with modular bipolar prosthesis. Balan et al. ${ }^{[10]}$ described $94 \%$ satisfactory outcome in intracapsular neck of femur fracture treated with modular bipolar prosthesis. Sakthivel et al. ${ }^{[15]}$ showed $20 \%$ excellent results and $80 \%$ good results in a study of hip arthroplasty using bipolar prosthesis.

In this study according to Harris hip score results were $36.6 \%$ excellent, $43.3 \%$ good, $13.33 \%$ fair and $6.67 \%$ poor which was comparable to other studies.

In our study valgus malpositioning of the stem was found in 2 patients $(6.66 \%)$. There were no major postoperative complications like wound infection and other.

Sullivan et al. ${ }^{[17]}$ found $3.7 \%$ periprosthetic fracture Following Modular Hip Hemiarthroplasty out of 429 patients. Balan et al ${ }^{[10]}$ found no periprosthetic fracture out of 30 patient treated with modular bipolar prosthesis. In our study no periprosthetic fracture has occurred our study is not without its own shortcomings. Firstly, our duration of followup of is less in assessing the longevity and functional endurance of the prosthesis used and hence come to definitive conclusions. Secondly, we have not evaluated the degree of intra-prosthetic motion at the inner bearing at each follow -up. Such studies are indicated because there are claims that the motion at the inner bearing reduces over time and most prostheses behave as unipolar prostheses over a period of time.

\section{Conclusion}

Hemiarthroplasty with modular bipolar prosthesis for fracture neck of femur gave satisfactory functional outcome, provides better range of movement, freedom from pain and more rapid return to unassisted activity with an acceptable complication rate. The complications were the exception rather than the norm. In view of late presentation, the outcome was good with decreased pain, improved daily living and function. Satisfactory functional results, minimal incidence of major radiological complications and the biomechanical advantage that comes with modular bipolar prosthesis made it a dependable endoprosthesis in orthopaedic practice. A longer follow up, along with a larger sample population should be able to show the durability and overall patient satisfaction with the modular bipolar prosthesis.

\section{Reference}

1. Royal College of Physicians.National Hip Fracture Database Robertson GA et al. Hip hemi-arthroplasty for neck of femur fracture 243 2018;9(11): WJO|www.wjgnet.com(NHFD) annual report 2017. London: Royal College of Physicians, 2017

2. Burge R, Dawson-Hughes B, Solomon DH, Wong JB, King A, Tosteson A. Incidence and economic burden of osteoporosis-related Fractures in the United States, 20052025. J Bone Miner Res 2007;22:465-475 [PMID: 17144789 DOI: $10.1359 / \mathrm{jbmr} .061113$

3. Cooper C, Campion G, Melton LJ. 3rd. Hip fractures in the elderly: a world-wide projection. Osteoporos Int 1992;2:285-289 [PMID: 1421796 DOI: 10.1007/BF01623184]

4. Hagino H, Nakamura T, Fujiwara S, Oeki M, Okano T, Teshima R. Sequential Change in quality of life for patients with incident clinical fractures: a Prospective study. Osteoporos Int 2009;20:695-702

5. Zhang $\mathrm{BF}$, Wang $\mathrm{PF}$, Huang $\mathrm{H}$ et al. Interventions for treating displaced intracapsular femoral neck fractures in the elderly: a Bayesian network meta-analysis of randomized controlled trials. Sci Rep 2017;7(1):13103

6. Parker MJ, Khan RJ, Crawford J et al. Hemiarthroplasty versus internal fixation for displaced intracapsular hip fractures in elderly. A randomized trial of 455 patients. $\mathrm{J}$ Bone Joint Surg Br 2002;84(8):1150-1155

7. Malhotra R, Arya R, Bhan S. Bipolar hemiarthroplasty in Femur Neck Fracture. Archives of Orthopaedic and Trauma Surgery 1995;114(2):79-82.

8. Sud A, Sood LK. Bipolar hip replacement for displaced Femur Neck Fracture in elderly patients. Indian Journal of Orthopaedics 1998;32:270-271.

9. Wang F, Zhang H, Zhang Z, et al. Comparison of bipolar hemiarthroplasty and total hip arthroplasty for displaced femoral neck fractures in the healthy elderly: a metaanalysis. BMC Musculoskelet Disord 2015;16(1):229.

10. Balan B, Shetty SK, Shetty A. Displaced Intra-capsular Neck Femur Fractures in Elderly: Austin Moore's prosthesis or Cemented Modular Bipolar Prosthesis. International Archives of Integrated Medicine, 2016;3:287-96.

11. Ali M. Modular Unipolar or Bipolar Hemiarthroplasty. El-Minia Medical Bulletin 2007;18:246-53.

12. Bhandari M, Devereaux P, Swiontkowski MF. Internal Fixation Compared with Arthroplasty for Displaced Fractures of the Femoral Neck. The Journal of Bone and Joint Surgery 2003;85-A:1673-81.

13. Keating JF, Grant A, Masson M, Forbes JF. Randomized Comparison of Reduction and Fixation, Bipolar Hemiarthroplasty, and Total Hip Arthroplasty. The Journal of Bone and Joint Surgery 2006;88-A:249-60.

14. Özer D, Çimen O, Aykut ÜS. Cemented Versus Cementless Modular Head Partial Prostheses in Femoral Neck Fractures of Elderly Patients: Comparison of Early Functional Results. Journal of Academic Research in Medicine 2012;2:1-5.

15. Sakthivel RN, Subramanyam BP, Balakrishnan V. A Study of Hip Arthroplasty Using Bipolar Endo-prothesis for Fracture Neck of Femur. International Journal of Research in Medical Sciences 2016;4:465-71. 
16. Sandhu HS. Management of Fracture Neck of Femur. Indian Journal of Orthopaedics 2005;39:130-6.

17. Sullivan NP, Hughes AW, Halliday RL, Ward AL, Chesser TJ. Early Complications Following Cemented Modular Hip Hemiarthroplasty. The Open Orthopaedics Journal 2015;9:15-9. 